\title{
Hippocampal volume, function and connectivity in anorexia nervosa: a systematic scoping review protocol
}

\author{
Johanna Keeler ${ }^{1,2^{*}}$, Olivia Patsalos ${ }^{2}$, Sandrine Thuret ${ }^{3}$, Hubertus Himmerich ${ }^{2}$, Janet Treasure ${ }^{2}$
}

\author{
Author affiliations \\ ${ }^{1}$ School of Psychology, College of Life and Environmental Sciences, University of Exeter, Exeter, UK \\ ${ }^{2}$ Department of Psychological Medicine, Institute of Psychiatry, Psychology \& Neuroscience, King's \\ College London, London, UK \\ ${ }^{3}$ Department of Basic and Clinical Neuroscience, Institute of Psychiatry, Psychology and \\ Neuroscience, King's College London, UK
}

\section{Corresponding author*}

Johanna Keeler

Washington Singer Laboratories, Perry Road, the University of Exeter, Exeter, Devon, EX4 4QG

Email: johanna.keeler@hotmail.com

\section{Keywords}

anorexia nervosa, hippocampus, hippocampal function, hippocampal volume, systematic scoping review

\section{Registration details}

This protocol was registered with the Open Science Framework on 3 May 2020, which can be found at https://osf.io/b64m7/

\section{ABSTRACT}

\section{Introduction}

Anorexia Nervosa (AN) is a serious mental health condition, which commonly follows a chronic course, termed severe-enduring AN (SE-AN). Recent evidence suggests SE-AN may be underpinned by an interaction between genetic risk factors, the endocrinological, immune and metabolic systems and deficiencies in neural structures. One structure found to be implicated over the course of AN is the hippocampus, which similarly has shown to be affected in other psychiatric disorders. The hippocampus is involved in processes such as memory and learning, as well as in the regulation of food intake. The literature on the hippocampus and AN is relatively heterogeneous and no reviews of hippocampal integrity in AN have been conducted. We will conduct a systematic scoping review of hippocampal volume, function and connectivity, as well as molecular components associated with hippocampal neurogenesis, to assess the variability in the literature and the current consensus on the hippocampus in AN.

\section{Methods and analysis}

We will use methods based on the Joanna Briggs Institute scoping review methods manual. Studies of any design in populations of AN with outcomes pertaining to our inclusion criteria will be located using an electronic database search of MEDLINE (PubMed), Web Of Science and PsychINFO. Two reviewers will independently screen titles, abstracts and full-texts and chart data of eligible studies. Study characteristics will be summarised during data analysis. Reported outcomes will include main study findings and methodology. 


\section{INTRODUCTION}

Anorexia nervosa (AN) is a serious and persistent psychiatric disorder with an estimated lifetime prevalence of 2-4\% amongst females, who are most affected by the disorder (Smink et al., 2012). AN is characterised by disturbed body image and an intense fear of weight gain, leading to severe dietary restriction, other weight loss behaviours, and significantly low weight (American Psychiatric Association, 2013). The disorder is associated with high relapse rates, with approximately $30 \%$ of individuals having an illness duration of greater than 15 years (Støving, Andries, Brixen, Bilenberg \& Hørder, 2011) and only 31\% reaching recovery after 9 years (Eddy et al., 2017). Illness duration of over 7 years has previously been considered as a severe-enduring course of AN (SE-AN; Broomfield et al., 2017), although there is some dispute in what characterises SE-AN. A major contributor to the high rates of relapse and prevalence of SE-AN is that the factors contributing to the chronic phase of the illness are not yet clear. However, there have been recent advances in our understanding, with genetic studies indicating a complex link between genes, environmental and nutritional factors (Watson, 2019; Hubel, 2019; Himmerich et al., 2019). SE-AN may be underpinned by the interaction between genetic risk factors and secondary physiological alterations involving the immune, endocrine and metabolic systems and neural systems such as the prefrontal cortex, hypothalamus, nucleus accumbens and hippocampus (Duriez et al., 2019; Himmerich et al., 2019).

One outcome of AN is a reduction in both white matter (WM) and grey matter (GM) volume. GM reductions are marked in specific structures such as the cerebellum, hippocampus, insula, amygdala and the prefrontal and cingulate cortices (Monzon et al., 2017), which is correlated with illness duration (Fonville, Giampietro, Williams \& Simmons, 2013). One study has found that reductions in hippocampal and cerebellar volumes were predictive of worse outcomes (Seitz, Herpertz-Dahlmann \& Konrad, 2016). The hippocampus is a structure most often associated with memory and learning processes, and human and animal data suggests it plays an important role in regulating food intake (Stevenson \& Francis, 2017). Further, it is an area particularly vulnerable to malnutrition, due to its high levels of neurogenesis and subsequent increased metabolic rate. Hippocampal proliferation has been found to be reduced during periods of neuroinflammation, mediated by increases in various cytokines, hormones and growth factors (Ryan \& Nolan, 2016). A hyperactive HPA axis, marked in those experiencing chronic stress, is known to cause neuroinflammation (Munhoz et al., 2008). Indeed, the hippocampus appears to be implicated in chronic stress conditions such as childhood maltreatment (Lecei and van Winkel, 2020), treatment-resistant depression (Liu et al., 2017), obsessive compulsive disorder (Atmaca et al., 2008) and post-traumatic stress disorder (PTSD; Shin, Rauch \& Pitman, 2006).

There have been a number of systematic reviews and meta-analyses examining hippocampal integrity in disorders such as major depressive disorder (MDD), PTSD and borderline personality disorder (e.g. Sala et al., 2004; Childress et al., 2013; Campbell, Marriott, Nahmias \& MacQueen, 2004). These reviews largely find a reduced hippocampal volume in such disorders, whilst often considering immunological factors involved in the body's response to stress (Frodl \& O'Keane, 2013). By comparison, AN has received less attention in regard to investigation of the hippocampus, despite similar features of chronic stress being apparent in AN such as the hyperactivity of the HPA axis (Chami et al., 2019). Therefore, there are transdiagnostic similarities across disorders such as $\mathrm{OCD}, \mathrm{MDD}$ and $\mathrm{AN}$. At present, there have been no reviews of cognitive function, structural alterations and related molecular activity specific to the hippocampus in those with AN.

The primary aim of this paper is therefore to review the nature and extent of the available literature on hippocampal integrity in AN. The scoping review format is being utilised as means of a preliminary examination of the nature and extent of the available literature (Grant \& Booth, 2009). This will allow us to map the available evidence, particularly looking at the variation in study 
methodology and the current consensus of the role of the hippocampus in AN. This was decided as a more appropriate mode of review than a full systematic review, due to the research area being relatively new (Munn et al., 2018).

\section{Objectives}

This scoping review aims to review the existing literature on hippocampal integrity among people diagnosed with AN. Specifically, this paper aims to answer the following questions:

1) What are the characteristics of these studies (e.g. sample; design)?

2) What measurements of hippocampal function and volume are used?

3) Which functional impairments, if any, specific to the hippocampus have been identified in those with AN?

4) Is there a basis for volumetric and connectivity deficits in the hippocampus among those with AN?

5) Is there a basis for molecular abnormalities in those with AN?

\section{METHODS AND ANALYSIS}

\section{Study design}

We will use a systematic scoping review framework to review the existing literature on hippocampal integrity and function in anorexia nervosa. As this is an underdeveloped research area employing a large variance in methodology, measurements and sample, a systematic scoping framework was deemed appropriate. This review will be conducted following the guidelines for scoping reviews developed by the Joanna Briggs Institute (Peters et al., 2020).

\section{Protocol}

This protocol was created in accordance with the Preferred Reporting Items for Systematic Reviews and Meta-Analysis for Protocols (PRISMA-P; Moher et al., 2015) reporting guideline (online supplementary materials A). This protocol was registered with the Open Science Framework on 3 May 2020. If required, any protocol amendments necessary will be documented on this webpage. The review is will commence in May 2020 and to continue until August 2020. The final scoping review will follow the Preferred Reporting Items for Systematic Reviews and Meta-Analysis extension for Scoping Reviews (PRISMA-ScR; Tricco et al., 2018)

\section{Eligibility criteria}

The eligibility criteria for the included studies are based on the Population, Concept and Context framework (PCC) recommended in the Joanna Briggs Institute Reviewers' Manual (2015).

Population (P): Patient groups of individuals with a current or past diagnosis of Anorexia Nervosa as defined by the diagnostic criteria in the Diagnostic and Statistical Manual of Mental Disorders (American Psychiatric Association, 2013), or by the International Statistical Classification of Diseases (ICD; World Health Organisation, 2018) criteria will be eligible. Diagnosis should have been made using a validated diagnostic tool or through a clinician diagnosis. Participants with co-morbid psychiatric diagnoses will be included. There will be no BMI cut-off for inclusion in this study, in order to include populations with AN at varying clinical severities and at various stages of illness. Studies using animal samples will be excluded.

Concept (C): Hippocampal volume in those with AN may be conducted using a variety of neuroimaging methods such as positron emission tomography (PET) and magnetic resonance imaging (MRI). Volumetric measurement may include other neural areas, with hippocampal volume 
being one outcome. Functional impairment localised to the hippocampus is measured using methods using functional MRI (fMRI), which is further measured using cognitive tasks whereby the hippocampus is thought to be involved in performance. These are largely tasks that measure aspects of cognition such as memory and learning, which the hippocampus is thought to be involved in (e.g. the Doors and People Test, Baddeley et al., 1994; pattern recognition memory task, Sahakian et al., 1988). Impairment refers to deficits in measurements in comparison to a control sample or norm values. Molecular aspects being examined include measurable blood components that are implicated in hippocampal integrity and neurogenesis such as: pro-inflammatory cytokines (specifically, tumour necrosis factors, TNF; interleukins, IL; Borsini, Zunszain, Thuret \& Pariante, 2015) and circulating neuronal growth factors (brain-derived neurotrophic factor, BDNF; vascular endothelial growth factor A, VEGF-A).

Context (C): Outcomes and sources of evidence pertaining to any context (e.g. country, healthcare setting, race, gender) are eligible for inclusion.

As this is the first review of its kind in this population, there will be no restrictions on the date of publication. Trials from any country or setting will be eligible, although only studies published in English will be included. There will be no restriction on study methodology as this is included as an outcome parameter and due to an anticipated low volume of search results.

\section{Information sources and search strategy}

Two reviewers (J.K. and O.P.) will independently search databases. The literature will be searched using the electronic databases MEDLINE (PubMed), Web of Science and PsychINFO, which will be supplemented by additional hand searches through reference lists. Journals will be searched from time of inception until May 2020. Search terms will be 1) anorex* coupled with 2) hippocamp*, although we will manually scan titles and abstracts for related terms such as: limbic system, grey matter, fimbria fornix, dentate gyrus, neurogenesis and connectivity. A draft of the search strategy is available in online supplementary materials $B$.

\section{Source selection}

Identified papers will be imported into a Microsoft Excel spreadsheet template where duplicates will be removed. Further to this, titles and abstracts will be independently screened by two reviewers. Reviewers will assess whether the sources meet the eligibility criteria by screening titles and abstracts, followed by a full text analysis. Discussion will enable discrepancies to be resolved and two clinicians (H.H. and J.T.) will resolve any remaining conflicts. Reasons for study exclusion will be logged on the same spreadsheet. Where necessary, we will contact authors to establish eligibility.

\section{Data charting}

After selection, the following data will be charted: publication identifiers (e.g. journal, year, first author); country of origin; design; study objective; methodology; sample characteristics (e.g. age group, diagnosis, sample size); relevant findings. We will chart the following data for outcomes: definition of outcome, definition of meaningful change, statistical method, outcome measurement(s) used and whether they are categorised as primary, secondary or were unspecified.

Following data charting, the identified outcomes will be synthesised and grouped through assignment to the following thematic "outcome terms" using a data extraction template: hippocampal volume, neuropsychological outcomes and molecular outcomes. Additionally, we will chart the study methodology and outcome measurements used. As the main rationale for the scoping review, hippocampal function and volume are identified as the main outcomes for which data will be charted, with study methodology and molecular factors as additional outcomes. 
Outcome grouping and mapping will be performed in consultation with clinicians on the project (H.H. and J.T.).

\section{Risk of bias assessment}

As recommended in the Joanna Briggs Institute manual for scoping reviews and due to the expected heterogeneity across study design and outcomes, this review will not conduct risk of bias assessments of included sources.

\section{Synthesis of results}

Analysis of data will include quantitative measures of study and outcome characteristics (e.g. sample age, body mass index, gender, total number of outcomes, number of included papers, total number of measurement instruments per grouping, median number of outcomes per study/grouping). We will utilise tables, for example, to display the characteristics of the included studies and outcomes.

\section{ETHICS}

This scoping review does not require ethical approval.

\section{AUTHOR CONTRIBUTIONS}

JK drafted the manuscript. All authors reviewed and provided feedback on the study design and manuscript. All authors read and approved the protocol before submission.

\section{FUNDING STATEMENT}

Johanna Keeler will receive funding through a Medical Research Council stipend for the Doctoral Training Programme. Hubertus Himmerich has received salary support from the NIHR BRC at the South London and Maudsley NHS Foundation Trust (SLaM) and KCL. 


\section{REFERENCES}

American Psychiatric Association, \& American Psychiatric Association. (2013). Diagnostic and statistical manual of mental disorders: DSM-5.

Atmaca, M., Yildirim, H., Ozdemir, H., Ozler, S., Kara, B., Ozler, Z., Kanmaz, E., Mermi, O. \& Tezcan, E. (2008). Hippocampus and amygdalar volumes in patients with refractory obsessive-compulsive disorder. Progress in neuro-psychopharmacology and Biological Psychiatry, 32(5), 1283-1286.

Baddeley A. D., Emslie H., \& Nimmo-Smith I. (1994). The Doors and People Test: A test of visual and verbal recall and recognition. Bury-St-Edmunds, UK: Thames Valley Test Company.

Borsini, A., Zunszain, P. A., Thuret, S., \& Pariante, C. M. (2015). The role of inflammatory cytokines as key modulators of neurogenesis. Trends in neurosciences, 38(3), 145-157.

Broomfield, C., Stedal, K., Touyz, S., \& Rhodes, P. (2017). L abeling and defining severe and enduring anorexia nervosa: A systematic review and critical analysis. International Journal of Eating Disorders, 50(6), 611-623

Campbell, S., Marriott, M., Nahmias, C., \& MacQueen, G. M. (2004). Lower hippocampal volume in patients suffering from depression: a meta-analysis. American Journal of Psychiatry, 161(4), 598-607.

Chami, R., Monteleone, A. M., Treasure, J., \& Monteleone, P. (2019). Stress hormones and eating disorders. Molecular and cellular endocrinology, 497, 110349.

Childress, J. E., McDowell, E. J., Dalai, V. V. K., Bogale, S. R., Ramamurthy, C., Jawaid, A., ... \& Schulz, P. E. (2013). Hippocampal volumes in patients with chronic combat-related posttraumatic stress disorder: a systematic review. The Journal of neuropsychiatry and clinical neurosciences, 25(1), 12-25.

Duriez, P., Ramoz, N., Gorwood, P., Viltart, O., \& Tolle, V. (2019). A Metabolic Perspective on Reward Abnormalities in Anorexia Nervosa. Trends in Endocrinology \& Metabolism.

Eddy, K. T., Tabri, N., Thomas, J. J., Murray, H. B., Keshaviah, A., Hastings, E., ... \& Franko, D. L. (2017). Recovery From Anorexia Nervosa and Bulimia Nervosa at 22-Year Follow-Up. The Journal of clinical psychiatry, 78(2), 184-189.

Fonville, L., Giampietro, V., Williams, S. C. R., Simmons, A., \& Tchanturia, K. (2014). Alterations in brain structure in adults with anorexia nervosa and the impact of illness duration. Psychological Medicine, 44(9), 1965-1975.

Frodl, T., \& O'Keane, V. (2013). How does the brain deal with cumulative stress? A review with focus on developmental stress, HPA axis function and hippocampal structure in humans. Neurobiology of disease, 52, 24-37.

Grant, M. J., \& Booth, A. (2009). A typology of reviews: an analysis of 14 review types and associated methodologies. Health Information \& Libraries Journal, 26(2), 91-108.

Himmerich, H., Hotopf, M., Shetty, H., Schmidt, U., Treasure, J., Hayes, R. D., Stewart, R. \& Chang, C. K. (2019). Psychiatric comorbidity as a risk factor for mortality in people with anorexia nervosa. European archives of psychiatry and clinical neuroscience, 269(3), 351-359. 
Hübel, C., Marzi, S. J., Breen, G., \& Bulik, C. M. (2019). Epigenetics in eating disorders: a systematic review. Molecular psychiatry, 24(6), 901-915.

Lecei, A., \& van Winkel, R. (2019). Hippocampal pattern separation of emotional information determining risk or resilience in individuals exposed to childhood trauma: linking exposure to neurodevelopmental alterations and threat anticipation. Neuroscience \& Biobehavioral Reviews.

Liu, W., Ge, T., Leng, Y., Pan, Z., Fan, J., Yang, W., \& Cui, R. (2017). The role of neural plasticity in depression: from hippocampus to prefrontal cortex. Neural Plasticity, 2017.

Martin Monzon, B., Henderson, L. A., Madden, S., Macefield, V. G., Touyz, S., Kohn, M. R., Clarke, S., Foroughi, N. \& Hay, P. (2017). Grey matter volume in adolescents with anorexia nervosa and associated eating disorder symptoms. European Journal of Neuroscience, 46(7), 2297-2307.

Moher, D., Shamseer, L., Clarke, M., Ghersi, D., Liberati, A., Petticrew, M., Shekelle, P. \& Stewart, L. A. (2015). Preferred reporting items for systematic review and meta-analysis protocols (PRISMA-P) 2015 statement. Systematic reviews, 4(1), 1.

Munhoz, C. D., Garcia-Bueno, B., Madrigal, J. L. M., Lepsch, L. B., Scavone, C., \& Leza, J. C. (2008). Stressinduced neuroinflammation: mechanisms and new pharmacological targets. Brazilian Journal of Medical and Biological Research, 41(12), 1037-1046.

Munn, Z., Peters, M. D., Stern, C., Tufanaru, C., McArthur, A., \& Aromataris, E. (2018). Systematic review or scoping review? Guidance for authors when choosing between a systematic or scoping review approach. BMC medical research methodology, 18(1), 143.

Peters, M.D.J., Godfrey, C., McInerney, P., Munn, Z., Tricco, A.C., \& Khalil, H. Chapter 11: Scoping Reviews (2020 version). In: Aromataris E, Munn Z (Editors). Joanna Briggs Institute Reviewer's Manual, JBI, 2020. Available from https://reviewersmanual.joannabriggs.org/

Ryan, S. M., \& Nolan, Y. M. (2016). Neuroinflammation negatively affects adult hippocampal neurogenesis and cognition: can exercise compensate?. Neuroscience \& Biobehavioral Reviews, 61, 121-131.

Sahakian, B. J., Morris, R. G., Evenden, J. L., Heald, A., Levy, R., Philpot, M., \& Robbins, T. W. (1988). A comparative study of visuospatial memory and learning in Alzheimer-type dementia and Parkinson's disease. Brain, 111(3), 695-718.

Sala, M., Perez, J., Soloff, P., Di Nemi, S. U., Caverzasi, E., Soares, J. C., \& Brambilla, P. (2004). Stress and hippocampal abnormalities in psychiatric disorders. European Neuropsychopharmacology, 14(5), 393-405.

Seitz, J., Herpertz-Dahlmann, B., \& Konrad, K. (2016). Brain morphological changes in adolescent and adult patients with anorexia nervosa. Journal of Neural Transmission, 123(8), 949-959.

Shin, L. M., Rauch, S. L., \& Pitman, R. K. (2006). Amygdala, medial prefrontal cortex, and hippocampal function in PTSD. Annals of the New York Academy of Sciences, 1071(1), 67-79.

Smink, F. R., Van Hoeken, D., \& Hoek, H. W. (2012). Epidemiology of eating disorders: incidence, prevalence and mortality rates. Current psychiatry reports, 14(4), 406-414. 
Stevenson, R. J., \& Francis, H. M. (2017). The hippocampus and the regulation of human food intake. Psychological bulletin, 143(10), 1011.

Støving, R. K., Andries, A., Brixen, K., Bilenberg, N., \& Hørder, K. (2011). Gender differences in outcome of eating disorders: a retrospective cohort study. Psychiatry research, 186(2-3), 362-366.

Tricco, A. C., Lillie, E., Zarin, W., O'Brien, K. K., Colquhoun, H., Levac, D., ... \& Hempel, S. (2018). PRISMA extension for scoping reviews (PRISMA-SCR): checklist and explanation. Annals of internal medicine, 169(7), 467-473.

Watson, H. J., Yilmaz, Z., Thornton, L. M., Hübel, C., Coleman, J. R., Gaspar, H. A., ... \& Medland, S. E. (2019). Genome-wide association study identifies eight risk loci and implicates metabo-psychiatric origins for anorexia nervosa. Nature genetics, 51(8), 1207-1214.

World Health Organization. (2018). International classification of diseases for mortality and morbidity statistics (11th Revision). 